\title{
Clinical translation in the treatment of hepatocellular carcinoma following the introduction of contrast-enhanced ultrasonography with Sonazoid
}

\author{
ATSUSHI HIRAOKA ${ }^{1}$, MISA ICHIRYU ${ }^{1}$, NAYU TAZUYA ${ }^{1}$, HIRONORI OCHI $^{1}$, ATSUSHI TANABE ${ }^{1}$, \\ HIROMASA NAKAHARA $^{1}$, SATOSHI HIDAKA ${ }^{1}$, TAKAHIDE UEHARA ${ }^{1}$, SOICHI ICHIKAWA ${ }^{1}$, AKI HASEBE ${ }^{1}$, \\ YASUNAO MIYAMOTO ${ }^{1}$, TOMOYUKI NINOMIYA ${ }^{1}$, MASASHI HIROOKA ${ }^{2}$, MASANORI ABE ${ }^{2}$, \\ YOICHI HIASA ${ }^{2}$, BUNZO MATSUURA ${ }^{2}$, MORIKAZU ONJI ${ }^{2}$ and KOJIRO MICHITAKA ${ }^{1}$ \\ ${ }^{1}$ Department of Gastroenterology, Ehime Prefectural Central Hospital; ${ }^{2}$ Department of Gastroenterology \\ and Metabology, Ehime University Graduate School of Medicine, Ehime, Japan
}

Received April 16, 2009; Accepted August 6, 2009

DOI: 10.3892/ol_00000010

\begin{abstract}
Some hepatocellular carcinoma (HCC) nodules are detectable with dynamic computed tomography, but not by conventional B-mode ultrasonography (US). Contrast-enhanced US (CEUS) with Sonazoid, a new injectable contrast agent, has been used in Japan since January 2007. The primary advantage of this agent is the ability to maintain observations continuously in the Kupffer phase. We assessed the clinical role of CEUS with Sonazoid for radiofrequency ablation (RFA). From January 2005 to December 2008, 1142 patients were treated with surgical resection, RFA, percutaneous ethanol injection or transcatheter arterial chemoembolization, following the exclusion of those patients treated with chemotherapy or supportive care. The patients included in the study were divided into the pre-CEUS ( $n=451,2005$ and 2006) and post-CEUS ( $n=691$, 2007 and 2008) groups. Clinical background (e.g., etiology, Child-Pugh classification, tumor node metastasis stage, percentage of patients matched with Milan criteria and selected therapies) was compared between the two groups. In addition, naïve cases were compared between the groups. There were 130 naïve HCC cases in the pre-CEUS group and 171 in the post-CEUS group. Although there were no significant differences for clinical background, the percentage of RFA cases increased from $21(\mathrm{n}=95)$ to $32 \%(\mathrm{n}=219)$ and from $32(\mathrm{n}=41)$ to $52 \%(\mathrm{n}=89)$ for total and naïve subjects, respectively, after CEUS was introduced $(\mathrm{P}<0.01)$. In naïve cases treated with RFA, tumor numbers in the post-CEUS group were larger than those of the pre-CEUS group $(1.15 \pm 0.48$ vs. $1.40 \pm 0.67$; $\mathrm{P}<0.01)$. CEUS with Sonazoid, therefore, makes it possible
\end{abstract}

Correspondence to: Dr Atsushi Hiraoka, Ehime Prefectural Central Hospital, Kasuga-Cho 83, Matsuyama, Ehime 790-0024, Japan E-mail: hirage@m.ehime-u.ac.jp

Key words: Sonazoid, contrast-enhanced ultrasonography, radiofrequency ablation, hepatocellular carcinoma to perform RFA in a considerable number of HCC cases that would otherwise be invisible by conventional B-mode US.

\section{Introduction}

Radiofrequency ablation (RFA) is performed worldwide for patients with hepatocellular carcinoma (HCC) as a curative local therapy because of the low rates of morbidity and mortality, and the high level of efficacy (1-5). Developments in ultrasonography (US) and contrast-enhanced US (CEUS) agents for hepatic tumors have enabled the diagnosis of HCC in the early stage, as CEUS makes it easier to detect small HCC tumors that are invisible to B-mode US.

In January 2007, Sonazoid ${ }^{\circledR}$ (Perflubutane, Daiichi Sankyo Co., Ltd., Japan) was approved as a new CEUS agent in Japan (6). Hatanaka et al found that this contrast agent has higher sensitivity and accuracy for the diagnosis of hepatic malignancy (7). We reported on the diagnostic efficacy of CEUS with Sonazoid in cases of HCC which were $<2 \mathrm{~cm}$ (8). The primary characteristic of this agent is the ability to repeatedly obtain a continuous enhanced view in the Kupffer phase. Numata et al also noted that repeated and continuous scanning in the Kupffer phase using CEUS with Sonazoid allowed for the easy detection of target lesions during RFA procedures (9). Thus, this contrast enhancement agent is considered to have a positive effect on therapeutic strategy. However, there are no known reports of changes in therapies prior to and following the introduction of CEUS with Sonazoid.

In order to evaluate the usefulness of Sonazoid as a treatment of patients with $\mathrm{HCC}$, we investigated the changes in therapies for the period before and after its introduction at our institution.

\section{Materials and methods}

Patients. This study included 1142 patients who were treated with surgical resection, RFA, percutaneous ethanol injection (PEIT) (10) or transcatheter arterial chemoembolization (TACE) $(11,12)$ from January 2005 to December 2008 at 


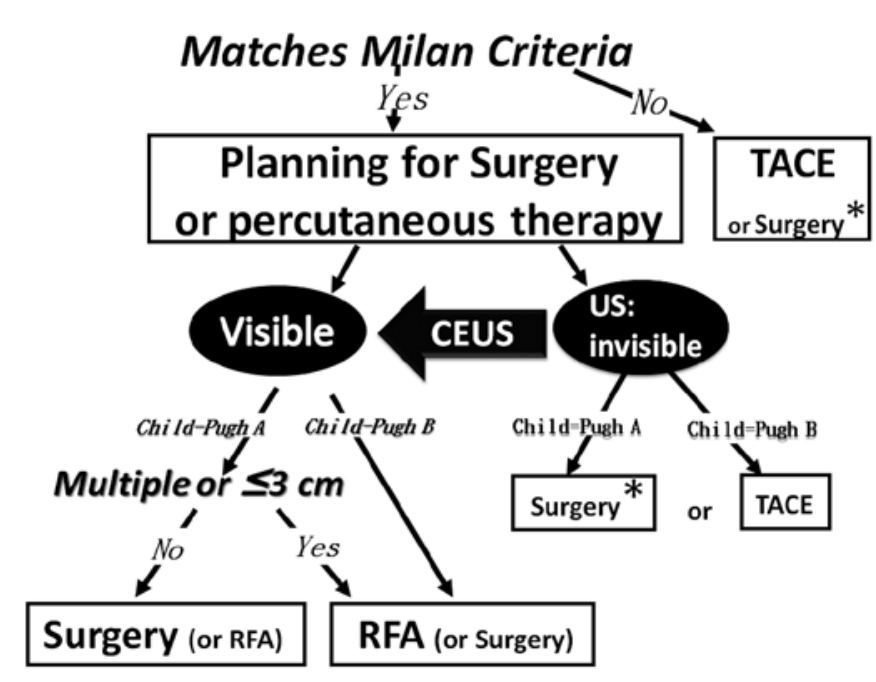

Figure 1. Strategy used at Ehime Prefectural Central Hospital for the treatment of patients with HCC matching Milan criteria. For cases shown to be invisible by conventional B-mode ultrasonography (US), we performed radiofrequency ablation therapy when target lesions were detected by contrast-enhanced US with Sonazoid. Surgical resection is considered, if possible (Milan criteria: single lesion $\leq 5 \mathrm{~cm}$ or $2-3$ lesions each $\leq 3 \mathrm{~cm}$ ).

our institution. Those treated with chemotherapy or supportive care were excluded. Patients were admitted to Ehime Prefectural Central Hospital and were treated based on our strategy (Fig. 1), which is based on established guidelines for the treatment of HCC in Japan (13). HCC diagnosis was based on histology, past history of HCC and/or cytological findings, or imaging evidence of tumor formation in the liver (with arterial hyper-vascularization) using at least 2 imaging modalities [US, dynamic computed tomography (CT), angiography and CT angio-portography (CTAP)] (14). Assistance with CEUS was considered in cases when the HCC tumors were visible by other modalities (e.g., dynamic CT and CTAP) and invisible by conventional B-mode US. RFA was performed when the target lesions were clearly visible by conventional B-mode US or CEUS with Sonazoid.

The patients were divided into 2 groups based on when CEUS with Sonazoid was introduced (pre-CEUS group, January 2005 to December 2006, $\mathrm{n}=451$; post-CEUS group, January 2007 to December 2008, n=691). The results and patient backgrounds were compared. In addition, the backgrounds of 130 naïve cases in the pre-CEUS group were investigated and compared to those of 171 naïve cases in the post-CEUS group. Clinical features, etiology, Child-Pugh classification (15), tumor node metastasis (TNM) stage (16), Japan Integrated Staging (JIS) score (17), percentage of patients matched with Milan criteria (single lesion $\leq 5 \mathrm{~cm}$ or $2-3$ lesions each $\leq 3 \mathrm{~cm}$ ) (18), and frequencies of each therapeutic modality selected (surgical resection, RFA, PEIT or TACE) were analyzed in the pre- and post-CEUS groups.

CEUS. Prior to CEUS, the SSD5500 machine(Aloka Co., Ltd., Tokyo, Japan), which had no program for CEUS was used to perform RFA. Aloka prosound $\alpha-10$ and EUB7500 (Hitachi Medical Corp., Tokyo, Japan) were then introduced in 2007.
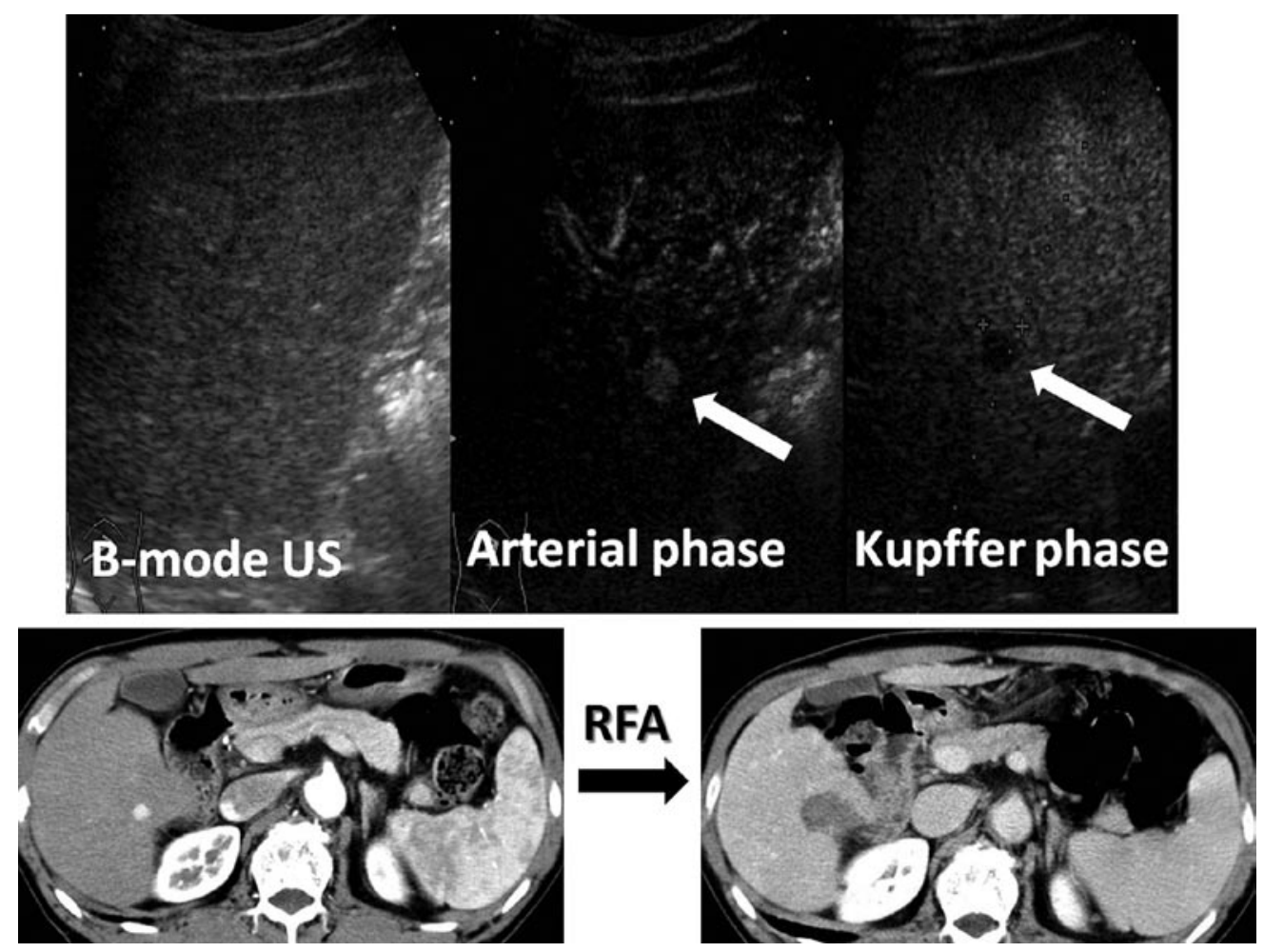

Figure 2. Representative case of small hepatocellular carcinoma $(0.9 \mathrm{~cm})$ in the 6 th segment that was visible by dynamic computed tomography $(\mathrm{CT})$ and invisible by conventional B-mode ultrasonography (US). With contrast-enhanced US with Sonazoid, the lesion was enhanced in the arterial phase and continuously shown as a defect in the Kupffer phase (white arrows). Radiofrequency ablation was successfully performed using imaging during the Kupffer phase. 
Table I. Background and frequency for 1142 patients with HCC.

\begin{tabular}{|c|c|c|c|}
\hline & Pre-CEUS group $(n=451)$ & Post-CEUS group $(n=691)$ & P-value \\
\hline $\begin{array}{l}\text { Etiology (HCV:HBV: } \\
\text { HBV+HCV:nonBnonC) }\end{array}$ & $355: 20: 14: 62$ & 534:45:12:100 & 0.619 \\
\hline Child-Pugh class (A:B:C) & 266:169:16 & $428: 238: 25$ & 0.452 \\
\hline TNM stage (I:II:III:IV) & $75: 177: 160: 39$ & $123: 250: 275: 43$ & 0.547 \\
\hline JIS score $(0: 1: 2: 3: 4: 5)$ & $46: 144: 142: 94: 21: 4$ & $84: 192: 243: 146: 23: 3$ & 0.414 \\
\hline $\begin{array}{l}\text { Milan criteria } \\
\text { (within:without) }\end{array}$ & $244: 207$ & $349: 342$ & 0.259 \\
\hline Frequency of RFA & $21 \%(n=95)$ & $32 \%(\mathrm{n}=219)$ & $<0.010$ \\
\hline RFA with CEUS & none & $18.7 \%(41 / 219)$ & \\
\hline
\end{tabular}

$\mathrm{HCC}$, hepatocellular carcinoma; HCV, hepatitis C virus; HBV, hepatitis B virus; $\mathrm{HBV}+\mathrm{HCV}$, double positive for $\mathrm{HBV}$ and $\mathrm{HCV}$; nonBnonC, double negative for HBV and HCV; TNM stage, tumor node metastasis stage; JIS score, Japan Integrated Staging score; RFA, radiofrequency ablation; CEUS, contrast-enhanced ultrasonography with Sonazoid. Milan criteria, single lesion $\leq 5 \mathrm{~cm}$ or $2-3$ lesions each $\leq 3 \mathrm{~cm}$.

Table II. Background and frequency for 301 patients with naïve HCC.

\begin{tabular}{|c|c|c|c|}
\hline & Pre-CEUS group $(n=130)$ & Post-CEUS group $(n=171)$ & $\mathrm{P}$-value \\
\hline $\begin{array}{l}\text { Etiology (HCV:HBV: } \\
\text { HBV+HCV:nonBnonC) }\end{array}$ & $88: 9: 3: 30$ & $125: 10: 0: 36$ & 0.371 \\
\hline Child-Pugh class (A:B:C) & $86: 41: 3$ & $119: 47: 5$ & 0.506 \\
\hline TNM stage (I:II:III:IV) & $27: 72: 20: 11$ & $40: 73: 51: 7$ & 0.545 \\
\hline JIS score $(0: 1: 2: 3: 4: 5)$ & $18: 58: 37: 9: 7: 1$ & $30: 60: 50: 28: 3: 0$ & 0.584 \\
\hline $\begin{array}{l}\text { Milan criteria } \\
\text { (within:without) }\end{array}$ & $94: 36$ & 119:52 & 0.700 \\
\hline Frequency of RFA & $32 \%(n=41)$ & $52 \%(\mathrm{n}=89)$ & $<0.010$ \\
\hline RFA with CEUS & none & $15.7 \%(14 / 89)$ & \\
\hline
\end{tabular}

$\mathrm{HCC}$, hepatocellular carcinoma; HCV, hepatitis C virus; HBV, hepatitis B virus; $\mathrm{HBV}+\mathrm{HCV}$, double positive for $\mathrm{HBV}$ and $\mathrm{HCV}$; nonBnonC, double negative for HBV and HCV; TNM stage, tumor node metastasis stage; JIS score, Japan Integrated Staging score; RFA, radiofrequency ablation; CEUS, contrast-enhanced ultrasonography with Sonazoid. Milan criteria, single lesion $\leq 5 \mathrm{~cm}$ or $2-3$ lesions each $\leq 3 \mathrm{~cm}$.

These machines have a program that allows them to perform CEUS with Sonazoid. Sonazoid was used as the CEUS agent $(0.5 \mathrm{ml} /$ body $)$ in all of the examinations. The target lesions were scanned following injection in the arterial and Kupffer phases. The arterial phase of CEUS imaging was defined as that which occurred from 10 to $60 \mathrm{sec}$ after injection of Sonazoid, and the Kupffer phase as that occurring 10 min after injection (19). Fifteen frames/sec were usually used to scan for a low mechanical index (0.2-0.3). A nodule was diagnosed as typical HCC when it was shown to be hypervascular in the arterial phase, and was revealed to be a defect lesion in the Kupffer phase (Fig. 2) by CEUS (20,21). In invisible cases,
RFA was performed using continuous imaging in the Kupffer phase of CEUS.

$R F A$. Prior to RFA treatment, $15 \mathrm{mg}$ of pentazocine hydrochloride and $25 \mathrm{mg}$ of hydroxyzine hydrochloride were administered intramuscularly. Local anesthesia was induced by $5 \mathrm{ml}$ of $1 \%$ lidocaine injected through the skin into the peritoneum along a predetermined puncture line. In cases with HCCs located near the lung, gallbladder or gastrointestinal tract, artificial pleural effusion (22) and artificial ascites were used as assistant methods for RFA. Midazolam (Dormicum ${ }^{\circledR}$, Astellas Pharma Inc., Japan) was injected intravenously at the 
Table III. Clinical features of naïve HCC patients treated with RFA.

\begin{tabular}{|c|c|c|c|}
\hline & Pre-CEUS group $(\mathrm{n}=41)$ & Post-CEUS group (n=89) & P-value \\
\hline Age (year) & $71.4 \pm 8.3$ & $69.0 \pm 9.2$ & 0.184 \\
\hline Gender (male:female) & $28: 13$ & $68: 21$ & 0.445 \\
\hline Tumor numbers & $1.15 \pm 0.48$ & $1.40 \pm 0.67$ & $<0.010$ \\
\hline Tumor diameter $(\mathrm{cm})$ & $2.15 \pm 0.7$ & $1.96 \pm 0.67$ & 0.848 \\
\hline Number of tumors $<1 \mathrm{~cm}$ & none & $7(8 \%)$ & 0.097 \\
\hline $\operatorname{AFP}(\mathrm{ng} / \mathrm{ml})$ & $84.8 \pm 243.8$ & $124.8 \pm 524.8$ & 0.423 \\
\hline AFP-L3 (\%) & $9.3 \pm 18.4$ & $6.5 \pm 13.3$ & 0.168 \\
\hline PIVKA-II (mAU/ml) & $71.1 \pm 99.0$ & $515.5 \pm 2224.3$ & 0.030 \\
\hline $\begin{array}{l}\text { Etiology (HCV:HBV: } \\
\text { HBV+HCV:nonBnonC) }\end{array}$ & $30: 2: 1: 8$ & $75: 5: 0: 9$ & 0.210 \\
\hline Child-Pugh class (A:B:C) & $27: 14: 0$ & $67: 22: 0$ & 0.192 \\
\hline TNM stage (I:II:III:IV) & $17: 21: 3: 0$ & $37: 35: 17: 0$ & 0.429 \\
\hline JIS score $(0: 1: 2: 3: 4: 5)$ & 11:19:11:0:0:0 & $30: 30: 24: 5: 0: 0$ & 0.850 \\
\hline
\end{tabular}

start of ablation $(0.1 \mathrm{mg} / \mathrm{kg})$. Most hypervascular nodules were subjected to TACE $(11,12)$ with epirubicin-lipiodol emulsion and multiporous gelatine particles $\left(\right.$ Gelpart $^{\circledR}$, Astellas Pharma Inc.) prior to RFA, for which we inserted a $20-\mathrm{cm}$ long 17-gauge radiofrequency electrode equipped with a 2- or 3-cm long exposed metallic tip (Radionics Cool-tip, Burlington, MA, USA).

Statistical analysis. Data are expressed as the mean \pm standard deviation. Statistical analyses were performed using Student's t-test for unpaired data and a Mann-Whitney U test as appropriate. Statistical analyses were performed using SPSS 16.0J (SPSS Japan Inc., Japan). P $<0.05$ was considered to be statistically significant.

\section{Results}

For the patients studied, there were no significant differences in the clinical backgrounds between the groups (Table I). HCC patient percentages, with the Milan criteria in the two groups, did not show a significant difference. However, the ratio of patients treated with RFA increased after CEUS was introduced [preCEUS vs. post-CEUS, 21 (95/451) vs. 32\% (219/691); $\mathrm{P}<0.01]$. Furthermore, of the patients treated with RFA in the post-CEUS group, $18.4 \%$ (41/219) received RFA with Sonazoid.

There were 130 naïve HCC patients in the pre-CEUS group and 171 in the post-CEUS group, with no significant differences in clinical background between the two groups (Table II). Naïve HCC patient percentages with the Milan criteria in the two groups were similar. However, the ratio of patients treated with RFA increased after CEUS was introduced [pre-CEUS vs. post-CEUS, 32 (41/130) vs. 52\% (89/171); P<0.01]. During the RFA procedure, assistance with CEUS was used in $15.7 \%$ (14/89) of naïve patients treated with RFA in the post-CEUS group. The clinical backgrounds of naïve HCC patients treated with RFA in the two groups were not significantly different, except for the number of tumors (pre-CEUS vs. post-CEUS, $1.15 \pm 0.48$ vs. $1.40 \pm 0.67 ; \mathrm{P}<0.01$; Table III). In addition, the percentage of tumors $<1 \mathrm{~cm}$ in diameter in naïve patients increased after the introduction of CEUS with Sonazoid, though the difference was not significant ( 0 vs. $8 \%$; $\mathrm{P}=0.097$ ).

\section{Discussion}

CEUS with Sonazoid, which is able to provide continuous imaging of target lesions in the Kupffer phase, has been reported to have a $94.7 \%$ rate of sensitivity and $81.8 \%$ rate of specificity for diagnosing small HCCs $(<2 \mathrm{~cm})$, while the positive and negative predictive values for those were 94.7 and $81.8 \%$, respectively (8). Minami et al reported that CEUS with Levovist ${ }^{\circledR}$ (Schering, Berlin, Germany) was useful as an indicator and guide for RFA (23). However, the combination of CEUS with another agent besides Sonazoid does not reveal the Kupffer phase continuously, because imaging during that phase is performed by bursting microbubbles that accumulate in Kupffer cells by sound waves (24). Continuous imaging in the Kupffer phase with Sonazoid occurs because the images are obtained with vibration rather than bursting microbubbles by sound waves, making it easy to observe abnormal areas continuously (9). Although RFA guided by CEUS with 
Sonazoid has been reported (9), there are no known reports of changes in therapies at any institution prior to and following the introduciton of this procedure.

No differences in clinical backgrounds, including the ratio of patients with Milan criteria, hepatic reserve function and TNM stage were noted between the groups in the present study. In contrast, the RFA ratio for 1142 subjects, as well as the naïve cases significantly increased after CEUS was introduced. We consider that these differences were attributable to continuous imaging in the Kupffer phase with Sonazoid, which enabled us to detect invisible HCCs by conventional B-mode US. Sonazoid allowed us to confirm that a greater number of target lesions were more visible by additional CEUS examination. As a result, we were able to perform the RFA procedure with confidence in the tumor location in those cases.

Livraghi et al reported that the same therapeutic effect was obtained after comparing patients, who had undergone surgical resection or RFA, with single HCC tumors $\leq 2.0 \mathrm{~cm}$ in diameter (25). We previously reported that RFA was safer and had a similar effect to surgical resection, though the number of patients with liver cirrhosis Child-Pugh B class treated with RFA was greater than that of patients treated with surgical resection (5). The results encouraged us to select RFA for liver cirrhosis patients with HCC. Sonazoid is a powerful agent for screening and performing RFA procedures for HCC nodules, which can be detected by other modalities such as dynamic CT but are difficult to detect by conventional B-mode US.

With the increasing number of small HCCs detectable by CEUS with Sonazoid, the number of patients that can potentially be treated with RFA will also increase, indicating that curative and low invasive RFA procedures will be performed in a larger number of HCC patients in the near future. We found that CEUS with Sonazoid allowed for the use of RFA for a considerable number of patients with HCCs, which would have otherwise been invisible by conventional B-mode US.

\section{References}

1. Rossi S, Di Stasi M, Buscarini E, et al: Percutaneous RF interstitial thermal ablation in the treatment of hepatic cancer. Am J Roentgenol 167: 759-768, 1996.

2. Tateishi R, Shiina S, Teratani $\mathrm{T}$, et al: Percutaneous radiofrequency ablation for hepatocellular carcinoma. An analysis of 1000 cases. Cancer 103: 1201-1209, 2005.

3. Shiina S, Teratani T, Obi S, Hamamura K, Koike Y and Omata M: Nonsurgical treatment of hepatocellular carcinoma: from percutaneous ethanol injection therapy and percutaneous microwave coagulation therapy to radiofrequency ablation. Oncology 62: 64-68, 2002.

4. Ng KK and Poon RT: Role of radiofrequency ablation for liver malignancies. Surg Practice 9: 94-103, 2005.

5. Hiraoka A, Horiike N, Yamashita Y, et al: Efficacy of radiofrequency ablation therapy compared to surgical resection in 164 patients in Japan with single hepatocellular carcinoma smaller than $3 \mathrm{~cm}$, along with report of complications. Hepatogastroenterology 55: 2171-2174, 2008.
6. Kindberg GM, Tolleshaug H, Roos N, et al: Hepatic clearance of Sonazoid perfluorobutane microbubbles by Kupffer cells does not reduce the ability of liver to phagocytose or degrade albumin microspheres. Cell Tissue Res 312: 49-54, 2003.

7. Hatanaka K, Kudo M, Minami Y and Maekawa K: Sonazoidenhanced ultrasonography for diagnosis of hepatic malignancies: comparison with contrast-enhanced CT. Oncology 75: 42-47, 2008.

8. Kan M, Horiike N, Hiraoka A, et al: Comparison diagnostic efficacy of contrast enhances ultrasonography with Perflubutane and dynamic computed tomography in patients with liver tumors smaller than $2 \mathrm{~cm}$. Hepatol Int 2: A255, 2008.

9. Numata K, Morimoto M, Ogura M, et al: Ablation therapy guided by contrast-enhanced sonography with Sonazoid for hepatocellular carcinoma lesions not detected by conventional sonography. J Ultrasound Med 27: 395-406, 2008.

10. Shiina S, Teratani T, Obi S, et al: Percutaneous ethanol injection therapy for liver tumors. Eur J Ultrasound 13: 95-106, 2001.

11. Takayasu K, Arii S, Ikai I, et al: Prospective cohort study of transarterial chemoembolization for unresectable hepatocellular carcinoma in 8510 patients. Gastroenterology 131: 461-469, 2006.

12. Hiraoka A, Kumagi T, Hirooka M, et al: Prognosis following transcatheter arterial embolization for 121 patients with unresectable hepatocellular carcinoma with or without a history of treatment. World J Gastroenterol 12: 2075-2079, 2006.

13. Makuuchi $\mathrm{M}$ and Kokudo $\mathrm{N}$ : Clinical practice guidelines for hepatocelluar carcinoma: the first evidence based guidelines from Japan. World J Gastroenterol 12: 828-829, 2006.

14. Bruix J and Sherman M: Management of hepatocellular carcinoma. Hepatology 42: 1208-1236, 2005.

15. Pugh RN, Murray-Lyon IM, Dawson JL, et al: Transection of the oesophagus for bleeding oesophageal varices. Br J Surg 60: 646-649, 1973.

16. Liver Cancer Study of Japan. The General Rules for the Clinical and Pathological Study of Primary Liver Cancer. 4th edition. Kanehara, Tokyo, p19, 2000.

17. Kudo M, Chung H, Haji S, et al: Validation of a new prognostic staging system for hepatocellular carcinoma: the JIS score compared with CLIP score. Hepatology 40: 1396-1405, 2004.

18. Mazzafero V, Regalia E, Doci R, et al: Liver transplantation for the treatment of small hepatocellular carcinomas in patients with cirrhosis. N Engl J Med 334: 693-699, 1996.

19. Watanabe R, Matsumura M, Chen CJ, et al: Characterization of tumor imaging with microbubble-based ultrasound contrast agent, Sonazoid, in rabbit liver. Biol Pharm Bull 28: 972-977, 2005.

20. Watanabe R, Matsumura M, Munemasa T, et al: Mechanism of hepatic parenchyma-specific contrast of microbubble-based contrast agent for ultrasonography: microscopic studies in rat liver. Invest Radiol 42: 643-651, 2007.

21. Kindberg GM, Tolleshaug H, Roos N, et al: Hepatic clearance of Sonazoid perfluorobutane microbubbles by Kupffer cells does not reduce the ability of liver to phagocytose or degrade albumin microspheres. Cell Tissue Res 312: 49-54, 2003.

22. Uehara T, Hirooka M, Ishida K, et al: Percutaneous ultrasoundguided radiofrequency ablation of hepatocellular carcinoma with artificially induced pleural effusion and ascites. J Gastroenterol 42: 306-311, 2007.

23. Minami Y, Kudo M, Kawasaki T, et al: Percutaneous radiofrequency ablation guided by contrast-enhanced harmonic sonography with artificial pleural effusion for hepatocellular carcinoma in the hepatic dome. Am J Roentgenol 182: 1224-1226, 2004.

24. Wang JH, Lu SN, Hung CH, et al: Small hepatic nodules $(\leq 2 \mathrm{~cm})$ in cirrhosis patients: characterization with contrast-enhanced ultrasonography. Liver Int 26: 928-934, 2006.

25. Livraghi T, Meloni F, Di Stasi M, et al: Sustained complete response and complications rates after radiofrequency ablation of very early hepatocellular carcinoma in cirrhosis: Is resection still the treatment of choice? Hepatology 47: 82-89, 2008. 